\title{
REVIEW ARTICLE OPEN Targeting IL-2: an unexpected effect in treating immunological diseases
}

\author{
Congxiu Ye ${ }^{1}$, David Brand ${ }^{2}$ and Song G. Zheng ${ }^{1,3}$
}

Regulatory T cells (Treg) play a crucial role in maintaining immune homeostasis since Treg dysfunction in both animals and humans is associated with multi-organ autoimmune and inflammatory disease. While IL-2 is generally considered to promote Tcell proliferation and enhance effector T-cell function, recent studies have demonstrated that treatments that utilize low-dose IL-2 unexpectedly induce immune tolerance and promote Treg development resulting in the suppression of unwanted immune responses and eventually leading to treatment of some autoimmune disorders. In the present review, we discuss the biology of IL-2 and its signaling to help define the key role played by IL-2 in the development and function of Treg cells. We also summarize proof-of-concept clinical trials which have shown that low-dose IL-2 can control autoimmune diseases safely and effectively by specifically expanding and activating Treg. However, future studies will be needed to validate a better and safer dosing strategy for low-dose IL-2 treatments utilizing well-controlled clinical trials. More studies will also be needed to validate the appropriate dose of IL-2/anti-cytokine or IL-2/anti-IL-2 complex in the experimental animal models before moving to the clinic.

Signal Transduction and Targeted Therapy (2018)3:2 https://doi.org/10.1038/s41392-017-0002-5

\section{INTRODUCTION}

Regulatory $\mathrm{T}$ cells (Treg) are crucial in maintaining immune homeostasis and prevention of autoimmune diseases. ${ }^{1,2}$ Treg cells are generally divided into two major subsets: naturally occurring, thymus-derived cells that react to self-antigens (Ags) and peripherally, or ex vivo induced, adaptive Treg cells that primarily respond to environmental $\mathrm{Ag}$ or in vitro stimulation with cytokine TGF- $\beta .^{3-7}$ Most of these cells are CD4 ${ }^{+}$cells that express CD25 (interleukin-2 (IL-2) receptor alpha chain) and the fork head box protein P3 (Foxp3) transcription factor that is required for their differentiation and function. ${ }^{8-11}$

When first discovered in 1976, IL-2 was characterized as a soluble factor with the unique ability to promote clonal expansion of $\mathrm{T}$ cells in vitro. ${ }^{12}$ It is primarily produced by antigen-activated $\mathrm{T}$ cells and can bind to three different high-affinity receptor subunits on target cell membranes. ${ }^{13}$ The discovery made by our group and others that IL-2 is a key cytokine for Treg cell differentiation, survival, and function ${ }^{14-17}$ has led to new opportunities for tipping the balance between Treg and effector T cells towards Tregs. Furthermore, clinical trials using low-dose IL2 have demonstrated that IL-2 shows great potential for expanding Treg cells and modulating immune pathologies. ${ }^{18-29}$

Here we review the current knowledge about IL-2 and its receptors, discuss the relationship between low-dose IL-2 and Treg development, especially focusing on mechanistic studies and clinical trials. These studies and trials have led to a shift in our understanding of IL-2 from a cytokine known for the activation of effector T cells against cancer when used at a high dose, to a cytokine that activates Treg cells to control autoimmunity at a low dose.

\section{IL-2 BIOLOGY}

IL-2, also named T-cell growth factor, was first discovered in 1976 and was characterized as a soluble factor with the unique ability to promote clonal expansion of T cells in vitro. ${ }^{12} \mathrm{IL}-2$ is a $15.5 \mathrm{kDa}$ four-bundle, a-helical protein member of the common cytokine receptor $\gamma$-chain family of cytokines (Fig. 1). First cloned in 1983, it has been the most highly investigated IL with a diverse role in the regulation of the immune system. ${ }^{30} \mathrm{IL}-2$ is predominantly produced by activated $\mathrm{CD}^{+} \mathrm{T}$ cells and, to a lesser extent by activated $\mathrm{CD}^{+}{ }^{\mathrm{T}}$ cells, activated dendritic cells, natural killer (NK) cells, NKT cells, as well as B cells. ${ }^{31}$

IL-2 exerts its pleiotropic biological activities in autocrine or paracrine fashion by binding to its receptors, which consists of three subunits including IL-2Ra(CD25), IL-2R $3(C D 122)$, and IL$2 R \gamma_{c}(C D 132){ }^{13}$ The heterotrimeric association of the IL-2Ra chain with IL-2R $\beta$ and IL-2R $\gamma_{c}$ provides a high-affinity receptor for IL- $2^{32}$. The heterodimeric association of the IL-2R 3 chain and the common $Y$ chain forms the intermediate-affinity IL-2R (binding affinity $\mathrm{Kd} \approx 1 \mathrm{nM}$ ), present in resting $T$ cells, memory $\mathrm{CD}^{+} \mathrm{T}$ cells and NK cells. ${ }^{14}$ By contrast, the IL-2RaßYc heterotrimeric complex, also known as the high-affinity IL-2R receptor $(\mathrm{Kd} \approx 10 \mathrm{pm})$, is transiently expressed by activated immune cells, including $T$ and $B$ lymphocytes, NK cells, and DCs even though it is constitutively expressed on Foxp3-expressing $\mathrm{CD}^{+}{ }^{\text {Tregs. }}{ }^{33}$ CD25 is a membrane protein with extensive $\mathrm{N}$ - and O-linked glycosylation that contains a short cytoplasmic tail lacking both kinase activity and phosphotyrosine motifs and which therefore does not have intrinsic signaling properties. The signaling function of the high-affinity IL-2 receptor is mediated through the $\beta$ and $\gamma$ chains. While these chains also lack kinase

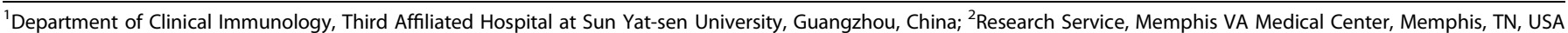
and ${ }^{3}$ Division of Rheumatology, Penn State Milton S. Hershey Medical Center, Hershey, PA, USA

Correspondence: Song G. Zheng (szheng1@pennstatehealth.psu.edu)
}

Received: 11 April 2017 Revised: 10 October 2017 Accepted: 30 October 2017

Published online: 19 January 2018 


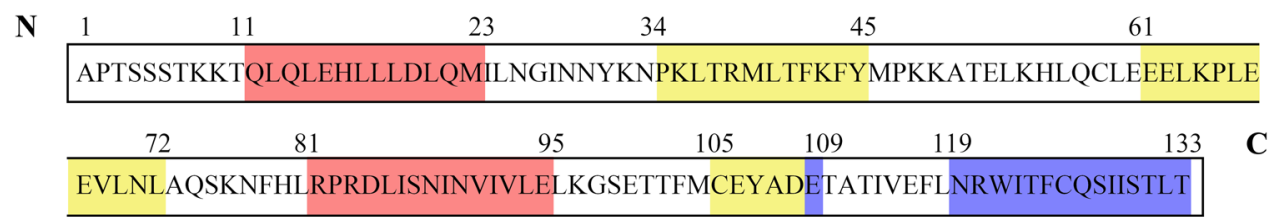

Fig. 1 Model structure of IL-2. Human IL-2 comprises 133 amino acids and weighs $15.5 \mathrm{kDa}$, while mouse IL-2 is comprised of 149 amino acids and weighs $16 \mathrm{kDa}$; they show $57 \%$ sequence homology. The amino acids that interact with IL-2R subunits are designated in yellow for IL-2R $\alpha$, red for IL-2R $\beta$ and blue for $\gamma_{c}$

activity, they constitutively bind to the Janus kinases JAK1 and JAK3. JAK1 and JAK3 kinase binding initiates phosphorylation of the $\beta$ and $\gamma$ chains, respectively, which then recruit STAT5a/ STAT5b via their $\mathrm{SH} 2$ domains. STAT5a/5b phosphorylation results in their translocation into the nucleus as a heterodimeric STAT complex, regulating gene transcription through binding to target DNA sequences. ${ }^{3,35}$ Alternatively, IL-2 signaling can induce the phosphorylation of the adaptor protein Shc which activates Ras-Raf MAP kinase and PI-3K pathways. ${ }^{36}$ Altogether, STAT5a/b, Ras-Raf MAP kinase and PI-3K signaling pathways allow rapid transmission of IL-2 signals from the membrane to the nucleus. These signals control, in a direct and indirect manner, the expression and function of multiple master regulator transcription factors implicated in several differentiation and immune regulatory pathways ${ }^{37}$ (Fig. 2).

IL-2 is a pleiotropic cytokine with myriad functions. Where Tregs are concerned, it supports their development in the thymus ${ }^{38}$ (Fig. 3), is a key survival factor for them in the periphery, and is required for their functional competence and stability ${ }^{17,39,40}$ (Fig. 3).

\section{IL-2 AND TREGS}

IL-2 is Involved in Treg Development, Stability, and Function Supporting the initial hypothesis that IL-2 is required for Treg development in vivo, work from Malek et al. ${ }^{41}$ demonstrated that the autoimmune disease expressed in $112 \mathrm{rb}^{-1-}$ mice could be prevented through transfer of $\mathrm{CD}_{2} 5^{+}$Tregs from WT mice into $\| 2 \mathrm{rb}^{-/-}$host mice. These studies demonstrated that $\| 2 \mathrm{rb}^{-/-}$mice lacked a functional population of Tregs. Additional work by this group demonstrated that thymus-specific expression of an $112 \mathrm{rb}$ transgene was sufficient to rescue the defect in Tregs development suggesting that the defect in $112 \mathrm{rb}^{-/-}$mice is due to a failure of Treg development in the absence of IL-2. ${ }^{42}$

Our research group made the first observation that IL-2 is important for the differentiation and function of $\mathrm{CD}^{+}{ }^{+} \mathrm{Foxp}^{+}$ Treg induced ex vivo. ${ }^{17}$ This finding was immediately validated by the laboratory of Dr. Ethan Shevach. ${ }^{43}$ Although TGF- $\beta$ and its receptor signal is essential for the Treg induction and development (induced Treg, iTreg) ${ }^{5}$ and its functional characteristics have been widely studied ${ }^{44-48}$, the role IL-2 plays in iTreg development, stability, and function is by no means redundant. ${ }^{17,43}$

\section{IL-2 signal plays a major role in thymic treg differentiation}

The importance of IL-2R signaling in thymic Treg differentiation is clearly demonstrated by the fact that the lethal autoimmunity in mice lacking $112 \mathrm{rb}$ is due to a failure to generate thymic Tregs, and this phenotype is completely restored by adoptive transfer of small numbers of wild-type Tregs. ${ }^{41}$ Moreover, retroviral transduction of $112 \mathrm{rb}^{-1-}$ bone marrow with wild-type $\| 2 \mathrm{rb}$, or a mutant construct capable of activating only STAT5 via Tyr-510, restored thymic Treg generation in bone marrow of chimeric mice. In contrast, restoration of Treg development did not occur when mutant constructs capable of activating RAS/PI3K, but not STAT5, were transduced into $112 \mathrm{rb}^{-1-}$ bone marrow cells and engrafted into recipient mice. ${ }^{49}$ Likewise, crossing $112 \mathrm{rb}^{-/-}$mice to transgenic mice expressing a constitutively active form of STAT5b (STAT5b-
CA mice)-restored Treg development in the thymus. ${ }^{49}$ Additional support for the role of STAT5 in Treg development came from two studies which demonstrated that conditional deletion of STAT5 in DP thymocytes (i.e., CD4-Cre $\times$ STAT5a/b ${ }^{\mathrm{FL} / F L}$ mice) had minimal effects on CD4SP thymocytes with the exception of CD4 ${ }^{+}$Foxp3 $^{+}$ thymic Treg. ${ }^{49,50}$ Altogether, these findings indicate that STAT5 activation on downstream of IL-2R is required for thymic Treg development.

\subsection{The IL-2 signal plays a major role in the homeostasis and} function of treg cells

There is an abundance of evidence demonstrating that the IL-2 signal plays a major role in the homeostasis and activation of $\mathrm{CD}^{+}$ CD25 ${ }^{+}$Foxp $^{+}$Tregs. $^{15}$ The quaternary complex consisting of IL-2 and IL-2Ra/ $\beta / \gamma$ induces the phosphorylation of STAT5, resulting in increased expression of CD25 and Foxp3 on Treg and activation of their suppressive activity. ${ }^{15,51}$ In the absence of IL-2 signaling, there was approximately a $50 \%$ reduction in the absolute numbers of Foxp3-expressing Treg cells in the thymic CD4 single-positive subset. ${ }^{39}$ The absolute numbers of Foxp $3^{+} \mathrm{T}$ cells in the periphery, however, were similar to that seen in wild-type mice, presumably because lymphopenia-induced lymphproliferation of the depleted thymic Treg pool was able to overcome the Treg developmental defect. $^{39}$ Another study followed the fate of a hemaglutininspecific monoclonal T-cell population in IL-2-deficient, HA/TCRdouble transgenic mice. The data suggested that IL-2/IL-2R signaling is necessary for the peripheral maintenance, but not development, of Foxp3 $^{+}$Treg cells. ${ }^{52}$ This conclusion was supported, in part, by the observations of another study reporting that IL-2/IL-2R signaling is required for Treg cell proliferation in vivo. ${ }^{53}$ In contrast, studies following the fate of HEL-specific $T$ cells in IL-2-deficient mice expressing HEL under control of an insulin promoter ${ }^{54}$ were more consistent with the idea that IL-2/IL$2 \mathrm{R}$ signaling is indispensable for development of Foxp ${ }^{+}$Treg cells. Takatoshi Chinen et al. ${ }^{55}$ found that the capture of IL-2 was dispensable for control of $\mathrm{CD}^{+} \mathrm{T}$ cells but was important for limiting the activation of $\mathrm{CD}^{+} \mathrm{T}$ cells, and that IL-2R-dependent activation of the transcription factor STAT5 had an essential role in the suppressor function of Treg cells separable from signaling via the T-cell antigen receptor by using genetic gain- and loss-offunction approaches. They hypothesized that genetic modification of Treg cells might hold promise for the optimal design of Treg cell-based therapies for a variety of autoimmune and inflammatory disorders and organ transplantation. When taken together, these observations in neo-antigen-specific TCR-transgenic mice suggest that differences in the levels and/or anatomical geography of antigen expression and/or in the affinities of the transgenic TCRs under study influence the extent with which IL-2/IL-2R deficiencies affect Treg development, peripheral homeostasis, or function. ${ }^{56}$

Notwithstanding these discrepancies, several lines of evidence support the idea that IL-2/IL-2R signaling is necessary for peripheral homeostasis of the $\mathrm{CD} 4^{+} \mathrm{CD} 25^{+}$Treg population. First, the peripheral $\mathrm{CD}^{+}{ }^{+} \mathrm{CD} 25^{+}$Treg pool is significantly reduced or absent in the absence of IL-2 or IL-2R..$^{39,52,53}$ Second, reconstitution of IL-2-competent hosts with a mixture of wild-type and IL-2Rdeficient bone marrow revealed that peripheral Treg arise 


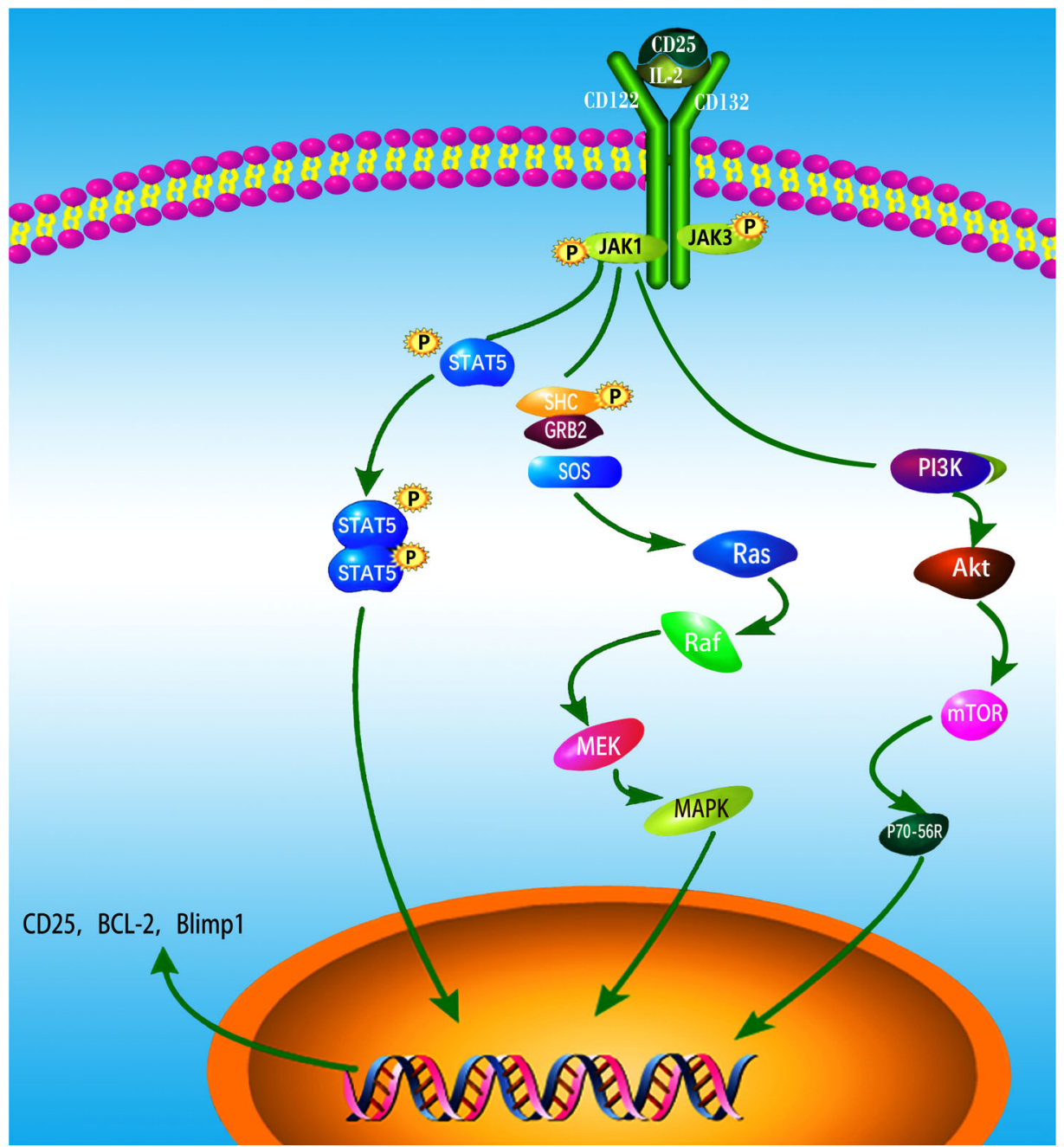

Fig. 2 IL-2 binding to IL-2 receptors (IL-2Rs) and downstream signaling. The high-affinity IL-2 receptor is comprised of CD25 (IL-2R $\alpha$ ), CD122 (IL-2R $\beta$ ), and CD132 (IL-2R $\gamma_{c}$ ). The signaling function of the high-affinity IL-2 receptor is mediated through IL-2R $\beta$ and $\gamma_{c}$ chains. These chains constitutively bind to JAK1 and JAK3 and phosphorylate which then recruit STAT5a/STAT5b via these molecules' SH2 domains. Phosphorylation of STAT5a/5b by JAKs results in the heterodimeric complex, which regulates gene transcription by binding to target DNA sequences. Phosphorylation of the adaptor SHC also leads to the activation of the Ras-Raf-MAPK and PI3K pathways. Any one or all of the STAT5a/b, Ras-Raf-MAPK, and PI3K signaling pathways can be activated under the appropriate situation. This accounts for IL-2's pleiotropic actions on target cells

exclusively from the wild-type donor marrow. ${ }^{57}$ Third, the size of the peripheral $\mathrm{CD} 4^{+} \mathrm{CD} 25^{+}$Treg population in neonatal mice was significantly reduced upon administration of neutralizing anti-IL-2 antibodies. ${ }^{52}$ Finally, treatment of NOD mice with a neutralizing anti-IL-2 antibody compromised Treg immune regulation and resulted in the development of accelerated T1D. ${ }^{58}$ Research has demonstrated that IL-2-deficient and IL-2R-deficient mice can be found to contain Foxp $3^{+} \mathrm{CD} 25^{-} \mathrm{T}$ cells which would suggest that Treg development is not IL-2 dependent. ${ }^{39,52}$ These cells, however, express lower levels of Foxp3 and represent immature, nonfunctional Treg cells. ${ }^{57}$ Yet another study along these lines found that $\mathrm{IL}-2^{-/-} \mathrm{Bim}^{-/-}$mice contain normal numbers of Foxp $3^{+}$cells in the periphery, but these cells also were not functional and the mice developed a lethal autoimmune phenotype. ${ }^{59}$

IL-2-deficient, IL-2R-deficient, or STAT5-deficient models and treg cells. In early phenotypic analyses of IL-2-deficient mice, Schorle and Sadlack found that lack of IL-2 does not lead to immunodeficiency or a failure of peripheral T cells to get activated but rather to hyper activation of the CD4 T-cell compartment. ${ }^{60,61}$ Very similar phenotypes were also seen in IL-2Rßand IL-2Ra deficient mice. ${ }^{62,63}$ Further research showed that deletion of IL-2 resulted in the absence of $\mathrm{CD}^{+} \mathrm{CD}_{2} 5^{+}$Tregs, which resulted in the occurrence of T-cell-mediated autoimmune diseases. ${ }^{58}$ Barmeyer et al. found that T cells and NK cells were present in neonatal mice with the deletion of IL-2. ${ }^{64}$ At 4 weeks, hyperplasia of lymph node, intestinal lymphoid tissue and splenomegaly occurred, at the same time the concentration of autoantibody in peripheral blood increased. ${ }^{64}$ At 9 weeks, $25-50 \%$ mice died of severe hemolytic anemia, while others suffered from severe inflammatory bowel diseases and other autoimmune phenotypes. ${ }^{64}$ Malek et al. ${ }^{65}$ also showed that the absence of Treg development in IL-2-and IL-2Rdeficient mice resulted in a lymphoproliferative autoimmune syndrome. Similarly, autoimmune disease development is linked to IL-2 deficiencies in humans ${ }^{66-68}$, most likely due to the expansion of immature non-functional Treg in the absence of IL2 signals. $^{69,70}$

Mice that lack STAT5a and STAT5b display massive lymphocyte infiltration of multiple organs. ${ }^{71}$ In these mice, there is a dramatic reduction in the size of the $\mathrm{CD}^{+} \mathrm{CD} 25^{+}$Treg population, resembling that seen in IL-2Ra-deficient mice. This is likely due to lack of Foxp3 expression, which requires STAT5, one of the key 
Thymus
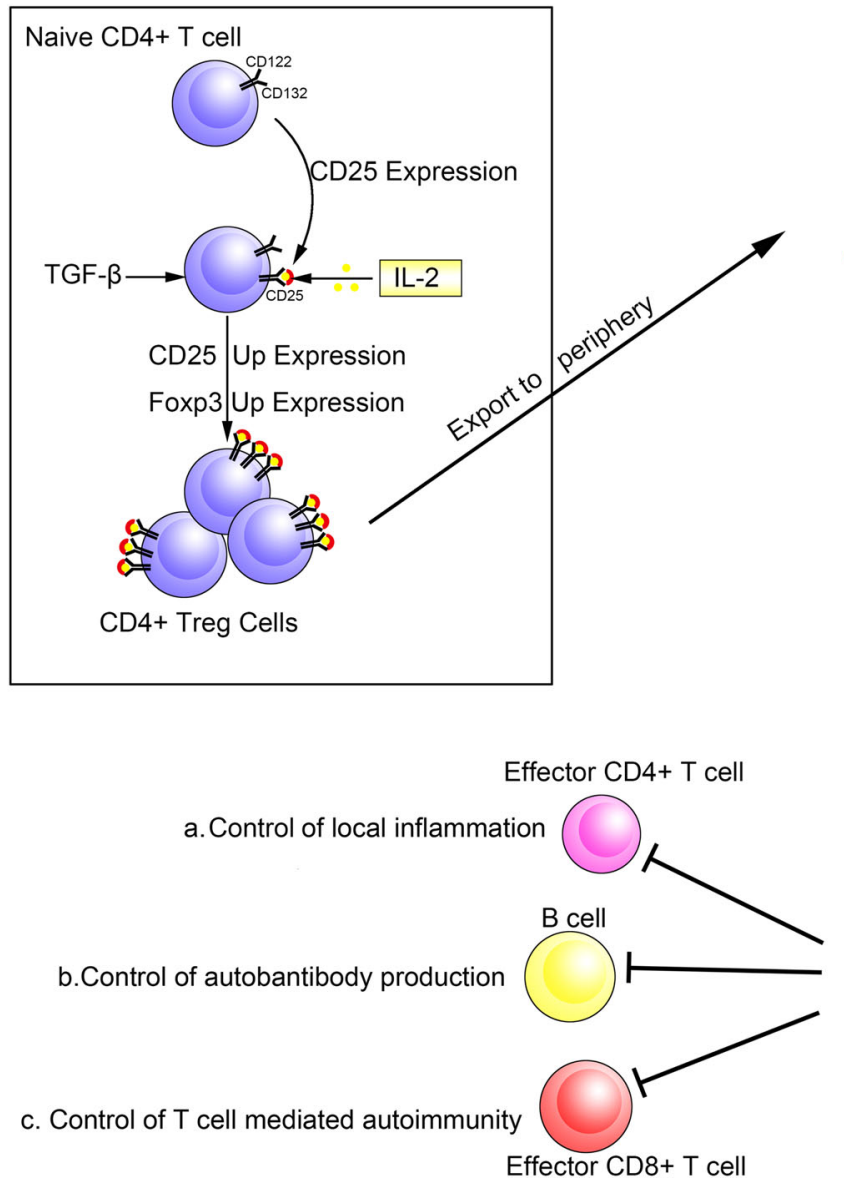

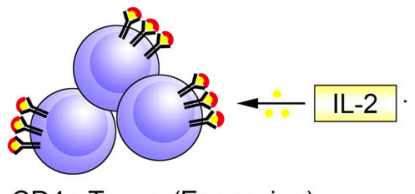

CD4+ Tregs (Expansion)

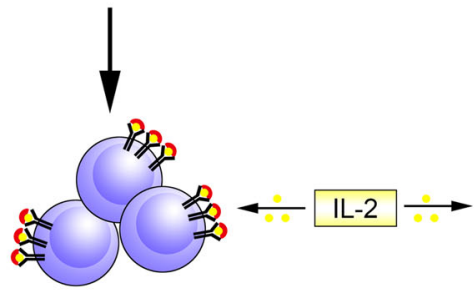

CD4+ Tregs (Homeostasis)
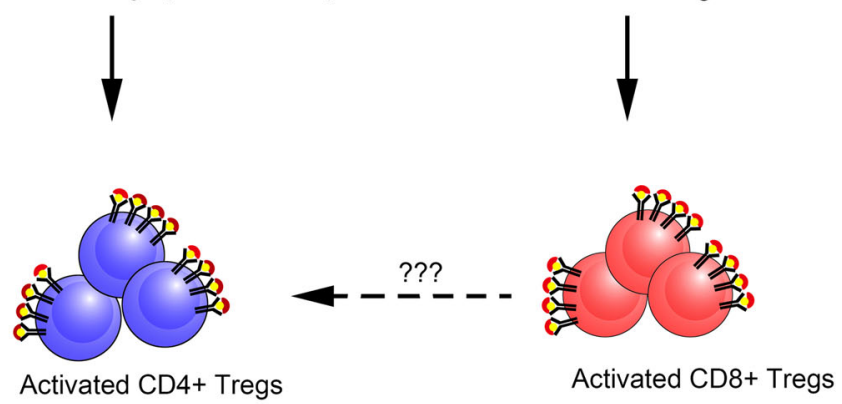

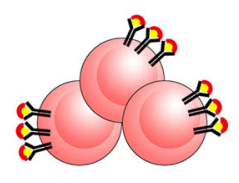

CD8+ Tregs

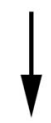

Fig. 3 Main role of IL-2 signal in thymus and in peripheral Tregs. In the thymus, naive CD4 ${ }^{+} \mathrm{T}$ cells transiently express CD25 and respond to IL2 through the STAT5s signaling pathway, and up regulate CD25 and Foxp3 expressions. Then, they begin to differentiation into mature CD4 ${ }^{+}$ Tregs. Mature $\mathrm{CD} 4^{+}$Tregs emigrate from the thymus and constitutively express CD25 and respond vigorously to IL-2 by up regulating CD25 and becoming activated $\mathrm{CD} 4^{+}$Tregs. $\mathrm{CD} 8^{+} \mathrm{T}$ cells also respond to IL-2 and become activated $\mathrm{CD} 8^{+}$Tregs. Activated CD4 ${ }^{+}$Tregs exert their suppressive functions to include $\mathbf{a}$ control of local inflammation; $\mathbf{b}$ control of autoantibody production; (c) control of T-cell-mediated autoimmunity. However, the mechanisms of the suppressive activity of $C D 8^{+}$Tregs in vivo remain to be determined

signal transduction molecules that connect IL-2 receptor activation to gene regulation in the nucleus. ${ }^{72,73}$

On the basis of these findings described above, it is likely that the appropriate low dose of IL-2 could be used to simulate Tregs and then in directly to reduce Th17 and Tfh activity. IL-2 might indeed become a prime candidate for the treatment of a broad range of autoimmune diseases.

Low-dose IL-2 treats diseases through expansion of treg cells. Base on the discovery that IL-2 is a key cytokine for Treg cell differentiation, survival and function, many studies tried to find out the therapeuticy potential in animal models and humans. Yenkel Grinberg-Bleyer et al. ${ }^{74}$ found that 5 days of low-dose IL-2 administration starting at the time of T1D onset can reverse established disease in NOD mice, with long-lasting effects by increasing the numbers of Treg cells in the pancreas and inducing expression of Treg related proteins including Foxp3, CD25, CTLA-4, ICOS, and GITR in these cells. However, Audrey Baeyens et al. ${ }^{75}$ observed that the RAPA/IL-2 combination had a failure to cure T1D. It is possible that RAPA counteracted low-dose IL-2 (25,000 IU daily) and then failed to control IL-2-boosted NK cells, and blocked IL-2-induced tolerance in a reversible way and was associated with an unexpected deleterious effect on glucosehomeostasis at multiple levels, including B-cell division, glucose tolerance, and liver glucose metabolism. In 2016,
Benjamin Bonnet et al. ${ }^{76}$ discovered that low-dose IL-2 (Id-IL-2)induced Treg expansion and activation that elicited protection against clinical manifestations of food allergy in two mouse models with OVA and peanut by evaluating the ability of Id-IL-2 to control allergy in an experimental model of food allergy, and the preventive and therapeutic effects were long-term effective over a 7-month period. Their further mechanistic studies indicated that protection from allergy could be explained by a Treg-dependent local modification of the Th1/Th2 balance and an inhibition of mast cell recruitment and activation. This study demonstrated that Id-IL-2 is efficient to prevent and to treat allergic immune responses, and thus represents a promising therapeutic strategy for managing allergic diseases for human in future.

Therapeutic use of IL-2 in humans was initially used at high doses. Ahmadzadeh et al. ${ }^{77}$ studied the impact of IL-2 administration on the frequency and function of human $\mathrm{CD}^{+}$ CD25 $5^{\text {hi }} \mathrm{T}$ cells in immune intact patients with melanoma or renal cancer, and found that administration of high-dose IL-2 increased the frequency of circulating CD4+ CD25hi Foxp3+ regulatory $\mathrm{T}$ cells, its suppressive activity and the expression of phenotypic markers associated with regulatory $\mathrm{T}$ cells, such as Foxp3. However, more and more clinical studies found that high-dose IL-2 has a poor safety profile but a robust efficacy in only a fraction of patients with a restricted set of 


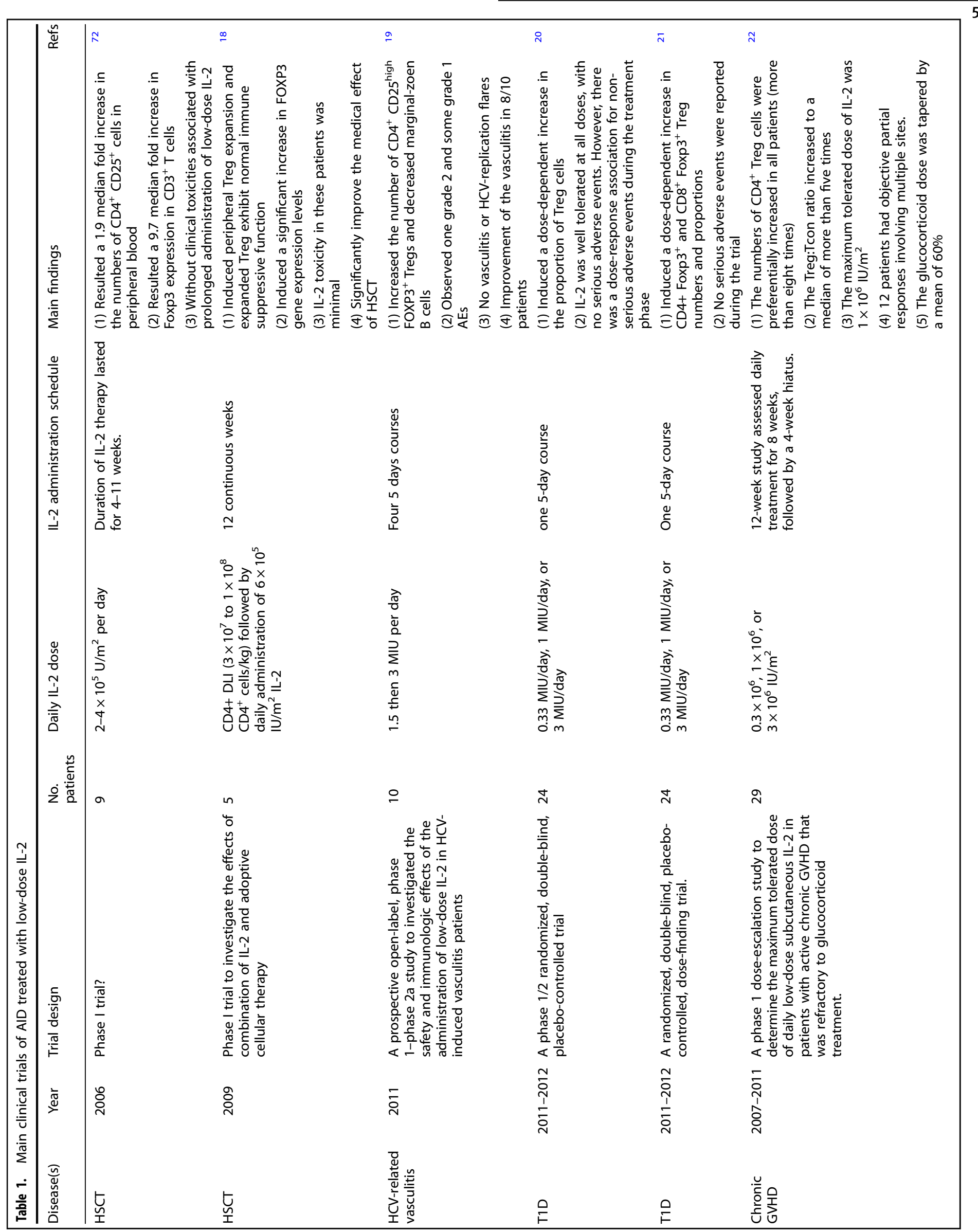




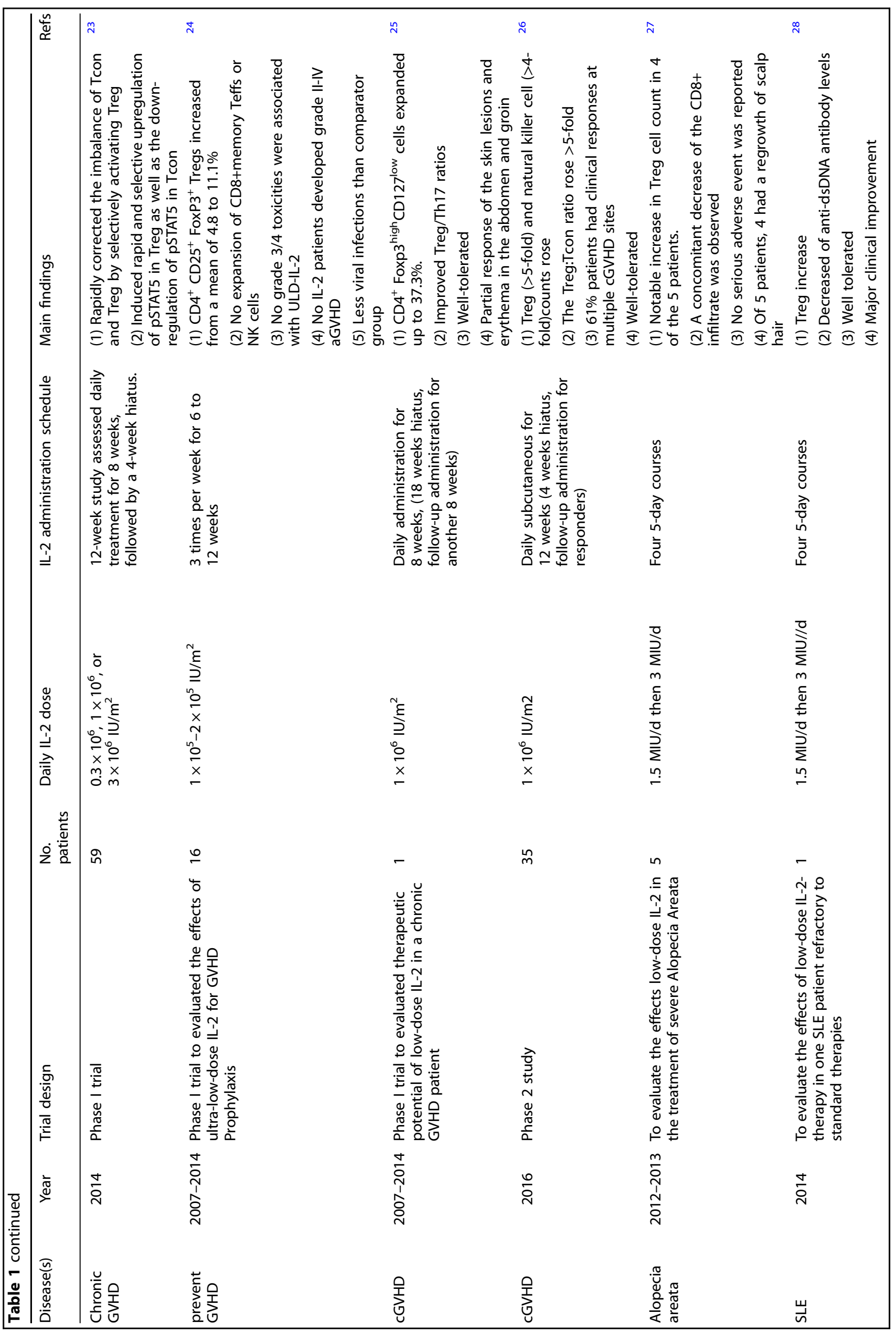


cancers. And the high toxicity severely restrained the use of highdose IL-2.

In recent years, it has been found that to avoid the severe side effects of high-dose IL-2 and also prevent, as far as possible, the activation of effector T cells, using low-dose IL-2 could be the solution. So, in this part, we summarize the clinical trials of lowdose IL-2 treatment in autoimmune diseases.

Low-dose IL-2 treatment and hematopoietic stem cell transplantation (HSCT)

Zorn et al. treated chronic myelogenous leukemia patients after allogeneic hematopoietic stem cell transplantation (HSCT) with low-dose IL-2 and found that treatment resulted in a 1.9 median fold increase in the numbers of $\mathrm{CD}^{+}{ }^{+} \mathrm{CD} 25^{+}$cells in peripheral blood as well as a 9.7 median fold increase in Foxp3 expression in $\mathrm{CD}^{+} \mathrm{T}$ cells, and that this effect appeared to increase as the treatment condition. ${ }^{72}$ In 2009 , the same group also found that the combination of low dose IL-2 with Treg infusion can significantly improve the clinical effect of HSCT by increasing the numbers of Treg cells in vivo. ${ }^{18}$

Low-dose IL-2 treatment and HCV-induced vasculitis Saadoun et al. ${ }^{19}$ evaluated the clinical efficacy and immunologic response after low-dose IL-2 therapy in HCV-related vasculitis patients, and found that treatment resulted in a 3 median folds increase of the numbers of $\mathrm{CD} 4^{+}$Treg cells and a 8 median folds increase of $\mathrm{CD}^{+}$Treg cells in peripheral blood, as well as a small increase in NK and CD56 ${ }^{\text {bright }}$ NK cells, but decrease of B cells.

Low-dose IL-2 treatment and T1D

An IL-2 dose determination study was performed in a small cohort of T1D patients in 2013. ${ }^{20}$ This randomized placebo-controlled phase I/II trial was under taken to define the lowest IL-2 dose that could induce Treg cells in 24 adult T1D patients randomized in groups of 6patients to either placebo or IL-2 at doses of $0.33,1$ or 3 million international units per day for a 5-day course. In this study, IL-2 therapy was safe and led to a dose-dependent increase in the numbers of $\mathrm{CD}^{+}$and $\mathrm{CD} 8^{+}$Treg cells with no increase or minimal increase in effector T cells and NK cells, and an absence of deleterious effects on insulin secretion. Rosenzwajg et al. ${ }^{21}$ also found that expansion of Treg cell populations was accompanied by a marked increase in their expression of activation markers such as CD25, glucocorticoid-induced TNFR-related protein (GITR) and cytotoxic $T$ lymphocyte antigen 4 (CTLA-4). However, both trials were not powered to assess effects on insulin secretion, so further clinical trials enrolling children and adults with recent onset T1D are needed. These studies will be undertaken in Europe (NCT02411253). ${ }^{78}$ Yu et al. ${ }^{79}$ found that IL-2 optimally stimulated tyrosine phosphorylated STAT5 at $~ 10$-fold lower levels of $\mathrm{IL}-2$ than $\mathrm{CD} 45 \mathrm{RO}^{+} \mathrm{CD}^{+}$memory $\mathrm{T}$ cells by quantifying the levels of initial proximal signaling and downstream gene activation.

Low-dose IL-2 treatment and chronic graft-versus-host disease (GVHD)

IL-2 was also shown to have beneficial effects for the treatment of chronic graft-versus-host disease (GVHD), an alloimmune inflammatory disease occurring after allogeneic haematopoietic stem cell transplantation. In a study conducted by Koreth et al. ${ }^{22}$, a cohort of 29 patients with chronic GVHD refractory to glucocorticoid therapy received rlL-2 at three dose levels $(300,000,1$ million and 3 million IU) daily for a period of eight weeks. The numbers of $\mathrm{CD}^{+}$Tregs increased about 8 folds in all patients, and approximately $50 \%$ of patients evaluated demonstrated an objective partial response. In another study undertaken to define the mechanisms of action of IL-2 therapy, Matsuoka et al. ${ }^{23}$ examined the immunologic effects of low-dose IL-2 treatment on homeostasis of $\mathrm{CD}^{+}{ }^{+} \mathrm{T}$-cell subsets after transplant and found that 
IL-2 therapy resulted in a selective increase of STAT5 phosphorylation in Tregs and a decrease of pSTAT5 in effector T cells. Over an eight-week period, IL-2 therapy induced a series of changes in Treg homeostasis including increased proliferation and thymic export and an enhanced resistance to apoptosis. ${ }^{23}$ Similarly, low-dose IL-2 administration has been tested in patients receiving hematopoietic stem cell transplantation in order to prevent GVHD. ${ }^{24}$ No adverse events of grade 3 or 4 nor any induction of GVHD were reported. In addition to an expansion of Tregs, viral infections were actually reduced in the low-dose IL-2 treated group compared to the control group. ${ }^{24}$ In this regard, two more studies have been recently completed in chronic GVHD patients. ${ }^{25,26}$ NayounKim et al. ${ }^{25}$ gave a chronic GVHD patient daily low-dose IL-2 treatment for 8 weeks followed by an 18 weeks hiatus and a follow-up administration for another 8 weeks, and demonstrated that low-dose IL-2 could induce a $37.3 \%$ expansion of Tregs leading to the improved Treg/Th17 ratios. In another phase II study ${ }^{26}, 35$ adults with steroid-refractory cGVHD received daily IL-2 treatments of 1 million IU for 12 weeks. Compared with pretreatment levels, Treg and NK cell counts increased over 5-folds and 4-folds, respectively, without significant change in conventional $\mathrm{CD}^{+} \mathrm{T}$ cell or $\mathrm{CD}^{+} \mathrm{T}$ cell numbers. $^{26}$

Low-dose IL-2 treatment and alopecia areata

In one study ${ }^{27}$, low-dose recombinant IL-2 induced a marked increase in the numbers of Treg cells accompanied by a marked decrease in the numbers of infiltrating $\mathrm{CD} 8^{+}$effector $\mathrm{T}$ cells in scalp biopsies of patients with autoimmune alopecia areata during and after IL-2 treatment.

Low-dose IL-2 treatment and systemic lupus erythematosus (SLE) In addition to the autoimmune diseases mentioned above, the potential treatment effect of low-dose IL-2 has been further strengthened by success in human clinical studies in systemic lupus erythematosus (SLE). In a study by Humrich et al. ${ }^{28}$, one patient with severe SLE refractory to standard therapy received low-dose IL-2 first at 1.5 MIU and then at $3 \mathrm{MIU}$ for 5-day each. They found that the numbers of Treg increased and the antidsDNA antibody levels decreased. To define the effects of lowdose recombinant human IL-2 (rhlL-2) treatment on regulatory and effector $\mathrm{CD}^{+}$T-cell subsets in patients with active SLE, He et al. ${ }^{29}$ gave 40 patients with active SLE2 MIU IL-2 every other day for 2 weeks followed by a 2-week hiatus, and demonstrated that low-dose IL-2 could both increase the numbers of $\mathrm{CD} 25^{\text {high }} \mathrm{C}$ D $127^{\text {low }}$ Treg cells and enhance their function, and that the ratio of $\mathrm{TFH}^{+} \mathrm{TH} 17$ cells/Treg cells fell significantly. In addition to clinical trial of low-dose IL-2 above mentioned, there are 14 ongoing clinical trials listed in www.clinicaltrial.org (Table 1) and two modified IL-2 patent applications have been granted (www. uspto.gov).

\section{CONCLUSION}

The data summarized in this review have indicated that IL-2 plays a key role in promoting the development, homeostasis and function of regulatory T cells though IL-2/STAT5 signals. Additionally, the independent clinical trials have also shown the safety of low-dose IL-2 treated in multiple autoimmune diseases. Moreover, these trials have provided preliminary indications of significant biological and clinical efficacy. We hypothesize that low-dose IL-2 may be a prime candidate therapy for other autoimmune diseases such as Rheumatoid Arthritis (RA) in which a deficiency in Treg cell number or function is present. However, these new therapeutic approaches present some undesirable adverse effects. Three potential ways to prevent these adverse effects include: (1) using IL-2/anti-IL-2 complexes to target $\mathrm{CD} 25^{-}$expressing cells and expand Treg without inducing IL-2-mediated expansion of autoreactive effector $\mathrm{T}_{\text {cells }}{ }^{80}$; (2) combining IL-2 administration with a blockade of cytokine pathways to promote Treg function and prevent Tfh cell development. ${ }^{80}$ For example, coadministration of low-dose IL-2 (or IL-2/anti-IL-2 complexes) together with anti-IL6R might synergize to promote Tregmediated suppression ${ }^{81}$ in the absence of effector T-cell activation. (3) Modifying IL-2. Bell et al. ${ }^{82}$ compared the clinically approved IL-2 molecule, Proleukin, with two engineered IL-2 molecules with long half-live sowing to their fusion in monovalent and bivalent stoichiometry to a non-FcRybinding human IgG1 and demonstrate that single ultra-low doses of IL-2 fusion proteins induce a prolonged state of in vivo activation that increases Tregs for an extended period of time similar to multiple-dose Proleukin. Therefore, additional well-controlled clinical trials are needed to validate improved and safer dosing strategies for low-dose IL-2 used in autoimmune diseases, but also future studies need to be done both in experimental animal models and later in clinics in order to validate IL-2/anti-cytokine therapies or IL-2/anti-IL-2 complex or IL-2 modification therapies as well.

\section{ACKNOWLEDGEMENTS}

This work was partly supported by National Key R\&D Program of China (2017YFA0105801), Zhujiang Innovative and Entrepreneurial Talent Team Award of Guangdong Province (2016 ZT 06S 252), Science and Technology Program of Guangzhou, China (Special Project on the Integration of Industry, Education and Research) (2015080 20060), the Project Supported by Guangdong Natural Science Foundation (2014A030308005), and General Program of National Natural Science Foundation of China (81671611).

\section{ADDITIONAL INFORMATION}

Conflict of interest: The authors declare that they have no conflict of interest.

\section{REFERENCES}

1. Sakaguchi, S. et al. Regulatory $T$ cells and immune tolerance. Cell 133, 775-87 (2008).

2. Lan, Q. et al. Induced Foxp3(+) regulatory T cells: a potential new weapon to treat autoimmune and inflammatory diseases? J. Mol. Cell. Biol. 4, 22-8 (2012).

3. Sakaguchi, S. Naturally arising Foxp3-expressing CD25+CD4+ regulatory T cells in immunological tolerance to self and non-self. Nat. Immunol. 6, 345-52 (2005).

4. Piccirillo, C. A. \& Shevach, E. M. Naturally-occurring CD4+CD25+ immunoregulatory $T$ cells: central players in the arena of peripheral tolerance. Semin. Immunol. 16, 81-8 (2004).

5. Zheng, S. G. et al. Generation ex vivo of TGF-beta-producing regulatory T cells from CD4+CD25- precursors. J. Immunol. 169, 4183-9 (2002).

6. Horwitz, D. A., Gray, J. D. \& Zheng, S. G. The potential of human regulatory T cells generated ex vivo as a treatment for lupus and other chronic inflammatory diseases. Arthritis Res. 4, 241-6 (2002).

7. Thompson, C. \& Powrie, F. Regulatory T cells. Curr. Opin. Pharmacol. 4, 408-14 (2004).

8. Yagi, H. et al. Crucial role of FOXP3 in the development and function of human CD25+CD4+ regulatory T cells. Int. Immunol. 16, 1643-56 (2004).

9. Fontenot, J. D., Gavin, M. A. \& Rudensky, A. Y. Foxp3 programs the development and function of CD4+CD25+ regulatory T cells. Nat. Immunol. 4, 330-6 (2003).

10. Khattri, R. et al. An essential role for Scurfin in CD4+CD25+ T regulatory cells. Nat. Immunol. 4, 337-42 (2003).

11. Zheng, S. G. et al. Natural and induced CD4+CD25+ cells educate CD4+CD25cells to develop suppressive activity: the role of IL-2, TGF-beta, and IL-10. J. Immunol. 172, 5213-21 (2004).

12. Morgan, D. A., Ruscetti, F. W. \& Gallo, R. Selective in vitro growth of T lymphocytes from normal human bone marrows. Science 193, 1007-8 (1976).

13. Minami, Y. et al. The IL-2 receptor complex: its structure, function, and target genes. Annu. Rev. Immunol. 11, 245-68 (1993).

14. Malek, T. R. The biology of interleukin-2. Annu. Rev. Immunol. 26, 453-79 (2008).

15. Boyman, O. \& Sprent, J. The role of interleukin-2 during homeostasis and activation of the immune system. Nat. Rev. Immunol. 12, 180-90 (2012).

16. Liao, W., Lin, J. X. \& Leonard, W. J. Interleukin-2 at the crossroads of effector responses, tolerance, and immunotherapy. Immunity 38, 13-25 (2013). 
17. Zheng, S. G. et al. IL-2 is essential for TGF-beta to convert naive CD4+CD25- cells to CD25+Foxp3+ regulatory T cells and for expansion of these cells. J. Immunol. 178, 2018-27 (2007).

18. Zorn, E. et al. Combined CD4+ donor lymphocyte infusion and low-dose recombinant IL-2 expand FOXP3+ regulatory T cells following allogeneic hematopoietic stem cell transplantation. Biol. Blood. Marrow Transplant. 15, 382-8 (2009).

19. Saadoun, D. et al. Regulatory T-cell responses to low-dose interleukin-2 in HCVinduced vasculitis. N. Engl. J. Med. 365, 2067-77 (2011)

20. Hartemann, A. et al. Low-dose interleukin 2 in patients with type 1 diabetes: a phase 1/2 randomised, double-blind, placebo-controlled trial. Lancet Diabetes Endocrinol. 1, 295-305 (2013).

21. Rosenzwajg, M. et al. Low-dose interleukin-2 fosters a dose-dependent regulatory T cell tuned milieu in T1D patients. J. Autoimmun. 58, 48-58 (2015).

22. Koreth, J. et al. Interleukin-2 and regulatory T cells in graft-versus-host disease. $N$. Engl. J. Med. 365, 2055-66 (2011).

23. Matsuoka, K. et al. Low-dose interleukin-2 therapy restores regulatory $\mathrm{T}$ cell homeostasis in patients with chronic graft-versus-host disease. Sci. Transl. Med. 5, 179ra43 (2013).

24. Kennedy-Nasser, A. A. et al. Ultra low-dose IL-2 for GVHD prophylaxis after allogeneic hematopoietic stem cell transplantation mediates expansion of regulatory T cells without diminishing antiviral and antileukemic activity. Clin. Cancer Res. 20, 2215-25 (2014)

25. Kim, N. et al. Therapeutic potential of low-dose IL-2 in a chronic GVHD patient by in vivo expansion of regulatory T cells. Cytokine 78, 22-6 (2016).

26. Koreth, J. et al. Efficacy, durability, and response predictors of low-dose interleukin-2 therapy for chronic graft-versus-host disease. Blood 128, 130-7 (2016).

27. Castela, E. et al. Effects of low-dose recombinant interleukin 2 to promote Tregulatory cells in alopecia areata. JAMA Dermatol. 150, 748-51 (2014).

28. Humrich, J. Y. et al. Rapid induction of clinical remission by low-dose interleukin-2 in a patient with refractory SLE. Ann. Rheum. Dis. 74, 791-2 (2015).

29. $\mathrm{He}$, J. et al. Low-dose interleukin-2 treatment selectively modulates CD4(+) T cell subsets in patients with systemic lupus erythematosus. Nat. Med. 22, 991-3 (2016).

30. Taniguchi, T. et al. Structure and expression of a cloned cDNA for human interleukin-2. 1983. Biotechnology 24, 304-9 (1992).

31. Harris, D. P. et al. Reciprocal regulation of polarized cytokine production by effector B and T cells. Nat. Immunol. 1, 475-82 (2000).

32. Kohm, A. P. et al. Cutting Edge: Anti-CD25 monoclonal antibody injection results in the functional inactivation, not depletion, of $C D 4+C D 25+T$ regulatory cells. J. Immunol. 176, 3301-5 (2006).

33. Sakaguchi, S. et al. Pillars article: immunologic self-tolerance maintained by activated T cells expressing IL-2 receptor alpha-chains (CD25). Breakdown of a single mechanism of self-tolerance causes various autoimmune diseases. J. Immunol. 186, 3808-21 (2011). J. Immunol. 1995.

34. O'Shea, J. J., Gadina, M. \& Schreiber, R. D. Cytokine signaling in 2002: new surprises in the Jak/Stat pathway. Cell 109, S121-31 (2002).

35. Cacalano, N. A. \& Johnston, J. A. Interleukin-2 signaling and inherited immunodeficiency. Am. J. Hum. Genet. 65, 287-93 (1999).

36. Brennan, P. et al. Phosphatidylinositol 3-kinase couples the interleukin-2 receptor to the cell cycle regulator E2F. Immunity 7, 679-89 (1997).

37. Arenas-Ramirez, N., Woytschak, J. \& Boyman, O. Interleukin-2: biology, design and application. Trends Immunol. 36, 763-77 (2015).

38. Vang, K. B. et al. IL-2, -7 , and -15 , but not thymic stromal lymphopoeitin redundantly govern $\mathrm{CD} 4+$ Foxp3+ regulatory $\mathrm{T}$ cell development. J. Immunol. 181, 3285-90 (2008).

39. Fontenot, J. D. et al. A function for interleukin 2 in Foxp3-expressing regulatory T cells. Nat. Immunol. 6, 1142-51 (2005).

40. Barron, L. et al. Cutting edge: mechanisms of IL-2-dependent maintenance of functional regulatory T cells. J. Immunol. 185, 6426-30 (2010).

41. Malek, T. R. et al. CD4 regulatory T cells prevent lethal autoimmunity in IL-2Rbetadeficient mice. Implications for the nonredundant function of IL-2. Immunity 17, 167-78 (2002).

42. Malek, T. R. et al. Normal lymphoid homeostasis and lack of lethal autoimmunity in mice containing mature $\mathrm{T}$ cells with severely impaired IL-2 receptors. J. Immunol. 164, 2905-14 (2000).

43. Davidson, T. S. et al. Cutting Edge: IL-2 is essential for TGF-beta-mediated induction of Foxp3+ T regulatory cells. J. Immunol. 178, 4022-6 (2007).

44. Zheng, S. G. et al. CD4+ and CD8+ regulatory T cells generated ex vivo with IL-2 and TGF-beta suppress a stimulatory graft-versus-host disease with a lupus-like syndrome. J. Immunol. 172, 1531-9 (2004).

45. Zheng, S. G. et al. TGF-beta requires CTLA-4 early after T cell activation to induce FoxP3 and generate adaptive CD4+CD25+ regulatory cells. J. Immunol. 176, 3321-9 (2006).
46. Zheng, S. G. et al. Transfer of regulatory T cells generated ex vivo modifies graft rejection through induction of tolerogenic CD4+CD25+ cells in the recipient. Int Immunol. 18, 279-89 (2006).

47. Zheng, S. G., Wang, J. \& Horwitz, D. A. Cutting edge: Foxp3+CD4+CD25+ regulatory T cells induced by IL-2 and TGF-beta are resistant to Th17 conversion by IL-6. J. Immunol. 180, 7112-6 (2008).

48. Zhou, X. et al. Therapeutic potential of TGF-beta-induced CD4(+) Foxp3(+) regulatory T cells in autoimmune diseases. Autoimmunity 44, 43-50 (2011).

49. Burchill, M. A. et al. IL-2 receptor beta-dependent STAT5 activation is required for the development of Foxp3+ regulatory T cells. J. Immunol. 178, 280-90 (2007).

50. Yao, Z. et al. Nonredundant roles for Stat $5 \mathrm{a} / \mathrm{b}$ in directly regulating Foxp3. Blood 109, 4368-75 (2007).

51. Wuest, T. Y. et al. The influence of IL-2 family cytokines on activation and function of naturally occurring regulatory T cells. J. Leukoc. Biol. 84, 973-80 (2008).

52. D'Cruz, L. M. \& Klein, L. Development and function of agonist-induced CD25 +Foxp3+ regulatory $\mathrm{T}$ cells in the absence of interleukin 2 signaling. Nat. Immunol. 6, 1152-9 (2005).

53. Bayer, A. L. et al. Essential role for interleukin-2 for CD4(+)CD25(+) T regulatory cell development during the neonatal period. J. Exp. Med. 201, 769-77 (2005).

54. Liston, A., Siggs, O. M. \& Goodnow, C. C. Tracing the action of IL-2 in tolerance to islet-specific antigen. Immunol. Cell. Biol. 85, 338-42 (2007).

55. Chinen, T. et al. An essential role for the IL-2 receptor in Treg cell function. Nat. Immunol. 17, 1322-1333 (2016).

56. Wang, J., Wicker, L. S. \& Santamaria, P. IL-2 and its high-affinity receptor: genetic control of immunoregulation and autoimmunity. Semin. Immunol. 21, 363-71 (2009).

57. Bayer, A. L., Yu, A. \& Malek, T. R. Function of the IL-2R for thymic and peripheral CD4+CD25+ Foxp3+ T regulatory cells. J. Immunol. 178, 4062-71 (2007).

58. Setoguchi, R. et al. Homeostatic maintenance of natural Foxp3(+) CD25(+) CD4(+) regulatory $T$ cells by interleukin (IL)- 2 and induction of autoimmune disease by IL2 neutralization. J. Exp. Med. 201, 723-35 (2005).

59. Chougnet, C. A. et al. A major role for Bim in regulatory T cell homeostasis. J. Immunol. 186, 156-63 (2011).

60. Schorle, $H$. et al. Development and function of $T$ cells in mice rendered interleukin-2 deficient by gene targeting. Nature 352, 621-4 (1991).

61. Sadlack, B. et al. Ulcerative colitis-like disease in mice with a disrupted interleukin-2 gene. Cell 75, 253-61 (1993).

62. Suzuki, H. et al. Deregulated T cell activation and autoimmunity in mice lacking interleukin-2 receptor beta. Science 268, 1472-6 (1995).

63. Willerford, D. M. et al. Interleukin-2 receptor alpha chain regulates the size and content of the peripheral lymphoid compartment. Immunity 3, 521-30 (1995).

64. Barmeyer, C. et al. The interleukin-2-deficient mouse model. Pathobiology 70 139-42 (2002).

65. Malek, T. R. \& Bayer, A. L. Tolerance, not immunity, crucially depends on IL-2. Nat. Rev. Immunol. 4, 665-74 (2004).

66. Sharfe, N. et al. Human immune disorder arising from mutation of the alpha chain of the interleukin-2 receptor. Proc. Natl Acad. Sci. USA 94, 3168-71 (1997).

67. Caudy, A. A. et al. CD25 deficiency causes an immune dysregulation, polyendocrinopathy, enteropathy, X-linked-like syndrome, and defective IL-10 expression from CD4 lymphocytes. J. Allergy Clin. Immunol. 119, $482-7$ (2007).

68. Roifman, C. M. Human IL-2 receptor alpha chain deficiency. Pediatr. Res. 48, 6-11 (2000).

69. Cheng, G. et al. IL-2R signaling is essential for functional maturation of regulatory T cells during thymic development. J. Immunol. 190, 1567-75 (2013).

70. Yuan, X., Cheng, G. \& Malek, T. R. The importance of regulatory T-cell heterogeneity in maintaining self-tolerance. Immunol. Rev. 259, 103-14 (2014).

71. Snow, J. W. et al. Loss of tolerance and autoimmunity affecting multiple organs in STAT5A/5B-deficient mice. J. Immunol. 171, 5042-50 (2003).

72. Zorn, E. et al. IL-2 regulates FOXP3 expression in human CD4+CD25+ regulatory $T$ cells through a STAT-dependent mechanism and induces the expansion of these cells in vivo. Blood 108, 1571-9 (2006).

73. Murawski, M. R. et al. Upregulation of Foxp3 expression in mouse and human Treg is IL-2/STAT5 dependent: implications for the NOD STAT5B mutation in diabetes pathogenesis. Ann. NY Acad. Sci. 1079, 198-204 (2006).

74. Grinberg-Bleyer, Y. et al. IL-2 reverses established type 1 diabetes in NOD mice by a local effect on pancreatic regulatory T cells. J. Exp. Med. 207, 1871-8 (2010).

75. Baeyens, A. et al. Limitations of IL-2 and rapamycin in immunotherapy of type 1 diabetes. Diabetes 62, 3120-31 (2013).

76. Bonnet, B. et al. Low-Dose IL-2 induces regulatory T cell-mediated control of experimental food allergy. J. Immunol. 2016, 188-98 (2016). 
77. Ahmadzadeh, M. \& Rosenberg, S. A. IL-2 administration increases CD4(+)CD25(hi) Foxp3(+) regulatory T cells in cancer patients. Blood 107, 2409-14 (2006).

78. Dwyer, C. J. et al. Promoting immune regulation in type 1 diabetes using lowdose interleukin-2. Curr. Diab. Rep. 16, 46 (2016).

79. $\mathrm{Yu}, \mathrm{A}$. et al. Selective IL-2 responsiveness of regulatory T cells through multiple intrinsic mechanisms supports the use of low-dose IL-2 therapy in type 1 diabetes. Diabetes 64, 2172-83 (2015).

80. Ballesteros-Tato, A. Beyond regulatory T cells: the potential role for IL-2 to deplete T-follicular helper cells and treat autoimmune diseases. Immunotherapy 6, 1207-20 (2014).

81. Pasare, C. \& Medzhitov, R. Toll pathway-dependent blockade of CD4+CD25+ T cell-mediated suppression by dendritic cells. Science 299, 1033-6 (2003).

82. Bell, C. J. M. et al. Sustained in vivo signaling by long-lived IL-2 induces prolonged increases of regulatory T cells. J. Autoimmun. 56, 66-80 (2015).
Open Access This article is licensed under a Creative Commons Attribution 4.0 International License, which permits use, sharing, adaptation, distribution and reproduction in any medium or format, as long as you give appropriate credit to the original author(s) and the source, provide a link to the Creative Commons license, and indicate if changes were made. The images or other third party material in this article are included in the article's Creative Commons license, unless indicated otherwise in a credit line to the material. If material is not included in the article's Creative Commons license and your intended use is not permitted by statutory regulation or exceeds the permitted use, you will need to obtain permission directly from the copyright holder. To view a copy of this license, visit http://creativecommons. org/licenses/by/4.0/.

(c) The Author(s) 2018 